\title{
Desarrollo de aceros fundidos para ser tratados térmicamente mediante carbo-austemperado
}

\author{
Development of steel castings to be heat treated by carbo-austempering \\ Oscar Ríos-Diez $^{1^{*} \quad \text { Claudia Patricia Serna Giraldo }}{ }^{1}$ \\ Ricardo Aristizábal-Sierra ${ }^{1}$
}

Recibido 8 de agosto de 2018, aceptado 16 de agosto de 2019

Received: August 8, 2018, Accepted: August 16, 2019

\begin{abstract}
RESUMEN
El carbo-austemperado es un tratamiento térmico mediante el cual la superficie de un acero de bajo carbono se carbura y luego durante el enfriamiento, se somete a un ciclo isotérmico con el objetivo de favorecer la formación de estructuras bainíticas en la capa carburada. En esta investigación se diseñaron dos aceros de bajo carbono susceptibles a ser carburados, los cuales se denominaron como A1 y A2. Como parte de la puesta a punto del tratamiento de carbo-austemperado, se establecieron las condiciones óptimas de los ciclos térmicos de homogenización, austenización y austemperado. Los aceros desarrollados se caracterizaron en cada una de las etapas mediante espectrometría de emisión óptica (EEO), microscopía óptica (MO), microscopía electrónica de barrido (MEB), difracción de rayos X (DRX) y ensayo de microdureza Vickers (HV). Los resultados obtenidos permiten entender la influencia de la composición química sobre el control de las microestructuras obtenidas después del ciclo de transformación isotérmica, ya que, con frecuencia, la elaboración a nivel industrial del acero involucra transformaciones en enfriamiento continuo y solo mediante un balance entre los efectos producidos por un adecuado tratamiento térmico, se obtienen resultados óptimos en la microestructura y las propiedades mecánicas derivadas de esta.
\end{abstract}

Palabras clave: Carbo-austemperado, transformación bainítica, aceros bajo carbono.

\begin{abstract}
Carbo-austempering is a heat treatment in which the surface of a steel is carburized and then quenched and hold at a temperature above the martensite start temperature, Ms, to obtain a bainitic structure in the carburized layer. In this research, two low carbon steels susceptible to carburizing were designed, the steels were named as A1 and A2. As part of the implementation of the carbo-austempering process, the optimal conditions for homogenization, austenization and austempering were established. The developed steels were characterized in each of the stages by optical emission spectrometry (OES), optical microscopy $(O M)$, scanning electron microscopy (SEM), X-ray diffraction (XRD) and Vickers microhardness test $(H V)$. The results allow to understand the influence of chemistry on the control of the microstructures after the isothermal transformation, since often at an industrial level the fabrication of these kind of steels involves continuous cooling transformations and only by means of a correct balance with the heat treatment parameters, optimal microstructures and mechanical properties can be achieved.
\end{abstract}

Keywords: Carbo-austempering, isothermal transformation, low-carbon steel.

\footnotetext{
1 Universidad de Antioquia, Facultad de Ingeniería, Departamento de Ingeniería de Materiales, Grupo de Investigaciones Pirometalúrgicas y de Materiales Gipimme. Medellín, Colombia. E-mail: eduardo.rios@udea.edu.co

* Autor de correspondencia: eduardo.rios@udea.edu.co
} 


\section{INTRODUCCIÓN}

Los aceros bainíticos libres de carburos (CFB, por sus siglas en inglés) han demostrado una mejor resistencia al desgaste y a la fatiga que los aceros convencionales, cuando se logra favorecer la formación de estructuras bainíticas en la capa carburada en vez de estructuras martensíticas. Se han reportado valores de dureza aproximada de 59HRC, resistencia a la tensión entre $1.1 \mathrm{GPa}$ y $2.2 \mathrm{GPa}$, resistencia a la compresión del orden de $3 \mathrm{GPa}$, límite de cedencia cercano a $1.2 \mathrm{GPa}$, y ductilidad entre $5 \%$ y $20 \%$ con resistencia a la fractura (KIC) del orden de 45-90MPa-m ${ }^{1 / 2}$ [1-7]. Esta combinación de propiedades excepcionales hace a los aceros carbo-austemperados superiores a los aceros cementados, con costos de producción equiparable o menores, con gran potencial de aplicación en las industrias del transporte, construcción, agrícola y militar, entre otras.

Las capas carburadas en las que predominan microestructuras bainíticas se obtienen mediante austenización seguida de austemperado a una temperatura que se encuentre entre la temperatura de inicio de la transformación bainítica, Bs, y la temperatura de inicio de la transformación martensítica, Ms [1]. Esta microestructura provee resistencias mecánicas excepcionales, que se derivan de la ausencia de carburos de hierro y de la escala de la estructura, lo cual es función de la temperatura a la que se realiza el tratamiento isotérmico y de la composición química del acero. La precipitación de los carburos durante la transformación bainítica se puede evitar aleando el acero con silicio, en cantidades de $1.5 \%$ en peso aproximadamente [8]. El silicio presenta una baja solubilidad en la cementita, lo cual produce un aumento local de la actividad del carbono. Como consecuencia, el flujo de carbono es reducido y se inhibe el desarrollo del embrión de cementita. El carbono que se expulsa como consecuencia de la formación de la ferrita bainítica, enriquece la austenita residual produciéndose así su estabilización hasta la temperatura ambiente [9]. El resultado es una microestructura formada por placas muy finas de ferrita bainítica separadas por regiones de austenita enriquecida en carbono de entre $20 \mathrm{~nm}$ y $40 \mathrm{~nm}$ [10]. Esta microestructura es ideal, pues existe la posibilidad de mejorar simultáneamente la resistencia y la tenacidad del acero. La resistencia procede principalmente del tamaño ultrafino de las placas de ferrita bainítica, mientras las láminas de austenita retenida al ser muy dúctiles y encontrarse dispersas entre las placas de ferrita bainítica mejoran las propiedades de alargamiento [11].

En ausencia de elementos que inhiban la precipitación de carburos, se pueden favorecer dos morfologías de bainita. La primera, denominada bainita superior, se obtiene por sostenimiento isotérmico a temperaturas por encima de $\operatorname{los} 300^{\circ} \mathrm{C}$ [12]. En este caso, la alta temperatura permite que el carbono se difunda completamente fuera de la ferrita bainítica, promoviendo la precipitación de la cementita en el límite entre las placas de ferrita en crecimiento. En el segundo caso, cuando la transformación isotérmica se realiza a temperaturas por debajo de $\operatorname{los} 300^{\circ} \mathrm{C}$, la difusión de carbono en la ferrita está muy limitada, por lo tanto, puesto que todo el carbono no puede difundirse fuera de la ferrita, se formarán pequeños precipitados de cementita dentro de los listones de la ferrita bainítica, esta microestructura se denomina bainita inferior [13].

El desarrollo de los aceros en los últimos años se ha centrado en la obtención de materiales con mayor resistencia mecánica, mayor ductilidad y mayor tenacidad a la fractura que permitan: (1) incrementar la resistencia al desgaste debido a las altas cargas en los componentes con altas capacidades, (2) incrementar la vida útil de la maquinaria y (3) incrementar la demanda para el diseño de equipos ergonómicos, ligeros y de bajo precio [14-16]. Es por esto que en este trabajo se planteó la posibilidad de producir dos aceros de bajo carbono fundidos, tratados termoquímicamente en su superficie mediante carbo-austemperado, con el objetivo de profundizar en el entendimiento de las relaciones entre la composición química, los parámetros de tratamiento térmico, la microestructura y las propiedades mecánicas de estos materiales.

\section{MATERIALES Y MÉTODOS}

Para el desarrollo de los aceros se utilizó como punto de partida la información encontrada en la literatura [17-21], la cual permitió establecer los intervalos de composición química de los elementos aleantes principales. A continuación, se procedió con el análisis teórico para la fabricación de los aceros y la realización de los tratamientos térmicos para la obtención de las microestructuras 
deseadas. Este análisis incluyó la elaboración de las curvas de Tiempo-Temperatura-Transformación (TTT) mediante el software de acceso libre de la Universidad de Cambridge Program MAP_STEEL_ MUCG83 [22]. Se hizo énfasis en la determinación de las temperaturas de transformación perlítica, bainítica y martensítica, además de los tiempos de transformación, con el fin de evitar transformaciones de carácter reconstructivo a ferrita proeutectoide y perlita. También, mediante el software se hicieron iteraciones de varias composiciones químicas con el fin conocer el efecto de los elementos aleantes sobre las curvas de los diagramas TTT. Las temperaturas de inicio de transformación bainítica (Bs) y martensítica (Ms) fueron también obtenidas mediante las ecuaciones de Trzaska (1) y Capdevilla (2) [23], respectivamente.

$$
\begin{gathered}
\mathrm{B}_{\mathrm{s}}\left({ }^{\circ} \mathrm{C}\right)=771-231,5(\% \mathrm{C})-69(\% \mathrm{Mn}) \\
-23(\% \mathrm{Si})-58,5(\% \mathrm{Cr})-31(\% \mathrm{Ni})- \\
55(\% \mathrm{Mo})-41(\% \mathrm{~V}) \\
\mathrm{M}_{\mathrm{s}}\left({ }^{\circ} \mathrm{K}\right)=764,2-302,6(\% \mathrm{C})-30,6(\% \mathrm{Mn}) \\
-16,6(\% \mathrm{Ni})-8.9(\% \mathrm{Cr})+2,4(\% \mathrm{Mo}) \\
-11,3(\% \mathrm{Cu})+8.58(\% \mathrm{Co})+7,4(\% \mathrm{~W}) \\
-14.5(\% \mathrm{Si})
\end{gathered}
$$

De acuerdo al estudio teórico inicial, se fabricaron dos aleaciones en un horno de inducción marca BEGO modelo 26300 con atmósfera controlada de argón y vaciado en coquilla metálica de cobre mediante centrifugación. Las composiciones químicas y los perfiles de carbono en la capa carburizada de ambos aceros, se determinaron mediante espectrometría de emisión óptica (EEO) en un equipo marca Bruker Magellan, modelo Q8.

Las muestras en estado as-cast se homogenizaron a $1000{ }^{\circ} \mathrm{C}$ durante 4 horas con el fin de obtener una microestructura y composición química uniformes. Luego las muestras se carburizaron en medio sólido, para lo cual se utilizó una mezcla de $90 \%$ de carbón vegetal y $10 \%$ de carbonato de bario, a $930{ }^{\circ} \mathrm{C}$ durante 5 horas, con enfriamiento en el horno. Las muestras carburizadas se austenizaron a $840^{\circ} \mathrm{C}$ durante 1 hora, tras lo cual se austemperaron en un baño de sales de composición 50\% nitrito de sodio - $50 \%$ nitrato de potasio a $270{ }^{\circ} \mathrm{C}$, con tiempos de sostenimiento de 4 y 16 horas. La temperatura y tiempos de austemperado se eligieron de acuerdo con las curvas TTT de los materiales seleccionados.
Las muestras obtenidas se prepararon mediante técnicas metalográficas tradicionales, según la norma ASTM E3 [24], el pulido final se realizó con alúmina de $0,3 \mu \mathrm{m}$. Para el revelado metalográfico de las micrografías ópticas en el acero A1 se utilizó el reactivo Nital $2 \%$, con el objetivo de facilitar la identificación de los carburos de cementita, tanto en la bainita como en la martensita formada. Para el acero A2 se utilizó reactivo Klemm1, de acuerdo con lo cual, las fases presentes se pueden diferenciar de la siguiente manera: ferrita bainítica de color azul, martensita café y austenita retenida blanco. El tamaño de grano se determinó mediante la norma ASTM E112-13 [25]. La fracción volumétrica de la bainita y la martensita en cada muestra se determinó bajo los lineamientos de la norma ASTM E562-11 [26], mediante el método de conteo de puntos. Para este propósito se analizaron 20 campos de visión con el objetivo de tener precisión de la medida reportada.

Se realizó además difracción de rayos X (DRX) con el objetivo de cuantificar la cantidad de austenita retenida. Para la selección de las variables del ensayo de DRX, se verificaron datos registrados en la literatura [27-29], con base en estos se decidió utilizar un ángulo $2 \theta$, entre $30^{\circ}$ y $100^{\circ}$, con un paso estándar inferior a 0,3 grados $/ \mathrm{min}$., la fuente empleada fue de cobalto $\left(K \alpha_{1}\right.$ de $\left.1.789 \AA\right)$. Por último, se realizaron barridos de microdureza desde la superficie hasta el núcleo de las muestras cada $100 \mu \mathrm{m}$, siguiendo los lineamientos de la norma ASTM E92-16 [30]. Se utilizó un microdurómetro SHIMADZU HMV-G 20DT con cargas de 100g-f y 15 segundos de sostenimiento, para cada muestra se realizaron dieciséis indentaciones, en un total de tres probetas por condición de ensayo.

\section{RESULTADOS Y ANÁLISIS}

Las temperaturas Bs y Ms determinadas por el programa MAP_STEEL_MUCG83 y calculadas con las ecuaciones empíricas de Trzaska (II) y Capdevilla, para los aceros desarrollados, se muestran en la Tabla 1. En la Figura 1, se muestran los diagramas TTT de los aceros A1 y A2, tanto para el núcleo $(0,23 \% \mathrm{C})$ como para la superficie $(0,8 \%$ de $\mathrm{C})$. Se observa que las aleaciones diseñadas tienen similar frente adifusional y sus temperaturas $B_{S}$ y $\mathrm{M}_{\mathrm{S}}$ presentan una dependencia del contenido de carbono, lo que lleva a tener diferencias en el frente difusional entre el núcleo y la superficie de 
Tabla 1. Temperaturas de inicio de transformación Bs y Ms para el núcleo y capa carbo-austemperada de los aceros M1 y M2.

\begin{tabular}{|c|c|c|c|c|}
\hline Inicio Transformación & A1-Núcleo $\left({ }^{\circ} \mathbf{C}\right)$ & A1-Capa $\left({ }^{\circ} \mathbf{C}\right)$ & A2-Núcleo $\left({ }^{\mathbf{}} \mathbf{C}\right)$ & A2-Capa $\left({ }^{\circ} \mathbf{C}\right)$ \\
\hline \multicolumn{5}{|c|}{ Utilizando programa MAP_STEEL_MUCG83 } \\
\hline Bs & 567 & 320 & 568 & 320 \\
\hline Ms & 417 & 167 & 418 & 170 \\
\hline \multicolumn{7}{|c|}{ Utilizando ecuaciones de Trzaska (II) para Bs y Capdevilla para Ms } \\
\hline Bs & 600 & 468 & 570 & 438 \\
\hline \multicolumn{7}{|c|}{} \\
\hline
\end{tabular}
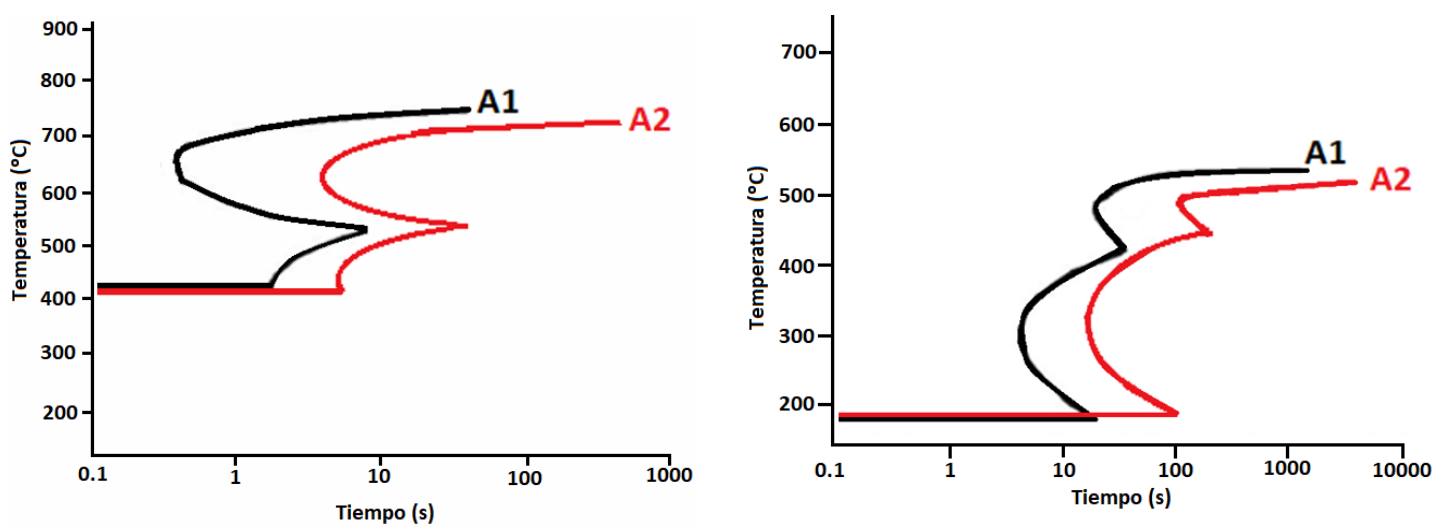

Figura 1. Diagramas TTT de comienzo de transformación para los aceros A1 y A2: a) Núcleo, b) capas carburizadas. Elaboradas con el programa MAP_STEEL_MUCG83.

cada acero, lo que está en correspondencia con los resultados arrojados por las ecuaciones de Trzaska y Capdevilla. Durante el enfriamiento de los aceros A1 y A2, especialmente en la superficie, y de acuerdo a los diagramas TTT, se evidencia que las transformaciones reconstructivas tienden a ocurrir a mayores tiempos, lo que favorece la ausencia de ferrita proeutectoide en la microestructura final de la capa carbo-austemperada de ambos aceros. Además, se nota que ambos aceros presentan una adecuada templabilidad bainítica, ya que los tiempos correspondientes a la curva $\mathrm{C}$ de las transformaciones displasivas son cortos, lo que evita la formación de martensita en el enfriamiento.

A partir, del análisis teórico de la viabilidad de la fabricación de los aceros y la obtención de las microestructuras deseadas, se procedió con el proceso de fundición. Los resultados de la composición química de los aceros fundidos, denominados A1 y A2 obtenida mediante EEO se muestran en la Tabla 2. La adición de cromo y manganeso se realizó
Tabla 2. Composición química de los aceros desarrollados, $\%$ en peso.

\begin{tabular}{|l|c|c|c|c|}
\hline Acero & $\begin{array}{c}\mathbf{C} \\
(\boldsymbol{\%} \mathbf{W})\end{array}$ & $\begin{array}{c}\mathbf{S i} \\
(\boldsymbol{\%} \mathbf{W})\end{array}$ & $\begin{array}{c}\mathbf{M n} \\
(\boldsymbol{\%} \mathbf{W})\end{array}$ & $\begin{array}{c}\mathbf{C r} \\
(\boldsymbol{\%} \mathbf{W})\end{array}$ \\
\hline $\mathrm{A} 1$ & 0,23 & 0,5 & 0,6 & 1,1 \\
\hline $\mathrm{A} 2$ & 0,23 & 1,8 & 0,6 & 1,1 \\
\hline
\end{tabular}

con el fin de dar mayor templabilidad a los aceros desarrollados. Asimismo, el cromo contribuye con el refinamiento de grano y al disolverse parcialmente en el hierro contribuye a mejorar la tenacidad. El manganeso mejora el endurecimiento por solución sólida que se pierde al tener un contenido tan bajo de carbono en el núcleo, además ayuda con el refinamiento de las agujas bainíticas [31]. Por último, la adición de silicio en el acero A2 se realizó con el objetivo de favorecer la presencia de austenita retenida libre de carburos de cementita, ya que retarda la precipitación de cementita durante la reacción bainítica, promoviendo la presencia 
de austenita retenida rica en carbono y estable a temperatura ambiente.

Las microestructuras en estado as-cast de los aceros A1 y A2 se presentan en las Figuras 2a y $2 \mathrm{~b}$ respectivamente. Ambas microestructuras corresponden a martensita con morfología en listones no homogénea y granos columnares, generada por la alta velocidad de enfriamiento a la que se sometieron las piezas durante el proceso de colada en la coquilla de cobre. Mediante el tratamiento de homogenización se buscó minimizar la segregación y los gradientes de concentración química, producto del proceso de solidificación y eliminar los granos columnares. El tratamiento de homogenización produjo una microestructura compuesta por ferrita proeutectoide poligonal (zonas de color blanco) y perlita (zonas de coloración oscura), las cuales son características de un acero hipoeutectoide. El tamaño de grano promedio de los aceros homogenizados fue de $38 \mu \mathrm{m}$ para el acero A1, Figura 2c, y $35 \mu \mathrm{m}$ para el acero A2, Figura 2d.

Posterior al tratamiento térmico de carburización en medio sólido en los aceros A1 y A2, se obtuvo una capa carburada con un contenido máximo en carbono de $0,8 \%$ y microestructura perlítica, como se observa en la Figura 3 para ambos aceros. Además, en la Figura 3 se observa el perfil de concentración de carbono a lo largo de la capa carburizada en ambos aceros, donde el espesor de capa promedio resultante fue de $941 \mu \mathrm{m} \pm 34 \mu \mathrm{m}$ para el acero A1 y $1046 \mu \mathrm{m} \pm 30 \mu \mathrm{m}$ para el acero A2.

En las Figuras 4, 5 y 6 se muestran las micrografías representativas de las probetas transformadas isotérmicamente para los aceros A1 y A2. En todas las muestras se encontraron bandas de martensita y bainita en la microestructura. La bainita formada en el acero A1, presenta una morfología en barras y no en placas (Figura 4). Además, en el acero A1, al tener un contenido de silicio inferior al límite necesario para inhibir la formación de cementita $(1,5 \%$ wt. $)$, la austenita no logra estabilizarse lo suficiente, y durante el enfriamiento hasta la temperatura ambiente transforma en martensita. De otra parte, como la estructura es demasiado fina para ser resoluble por microscopia óptica y la tonalidad de los carburos de cementita presentes, es muy similar a la martensita, se utilizó la microscopia
A1

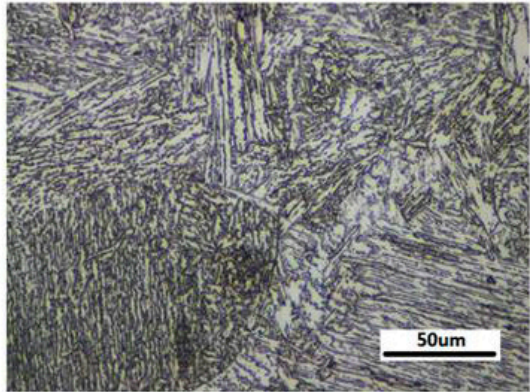

(a)

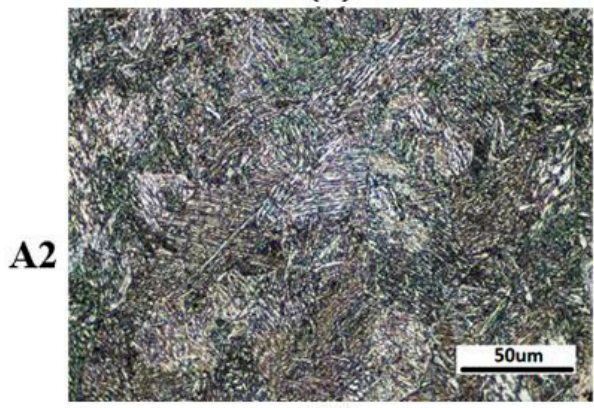

(b)

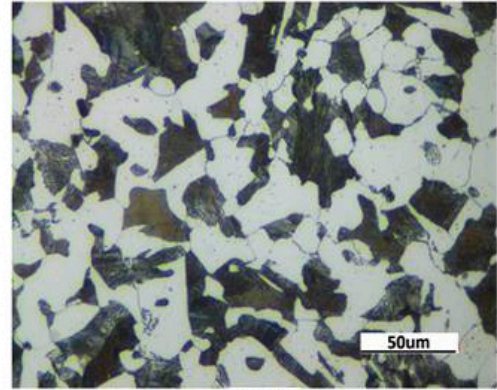

(c)

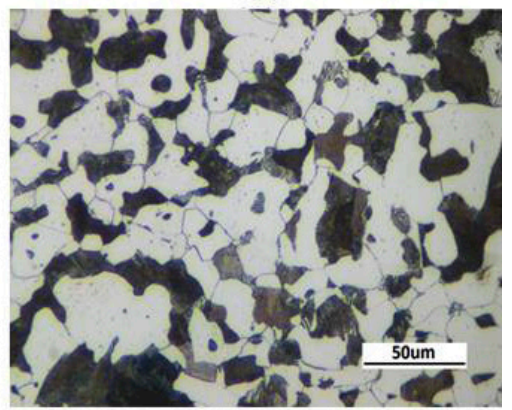

(d)

Figura 2. Estado as-cast son las micrografias (a) y (b) y las homogenizadas son la (c) y (d). 

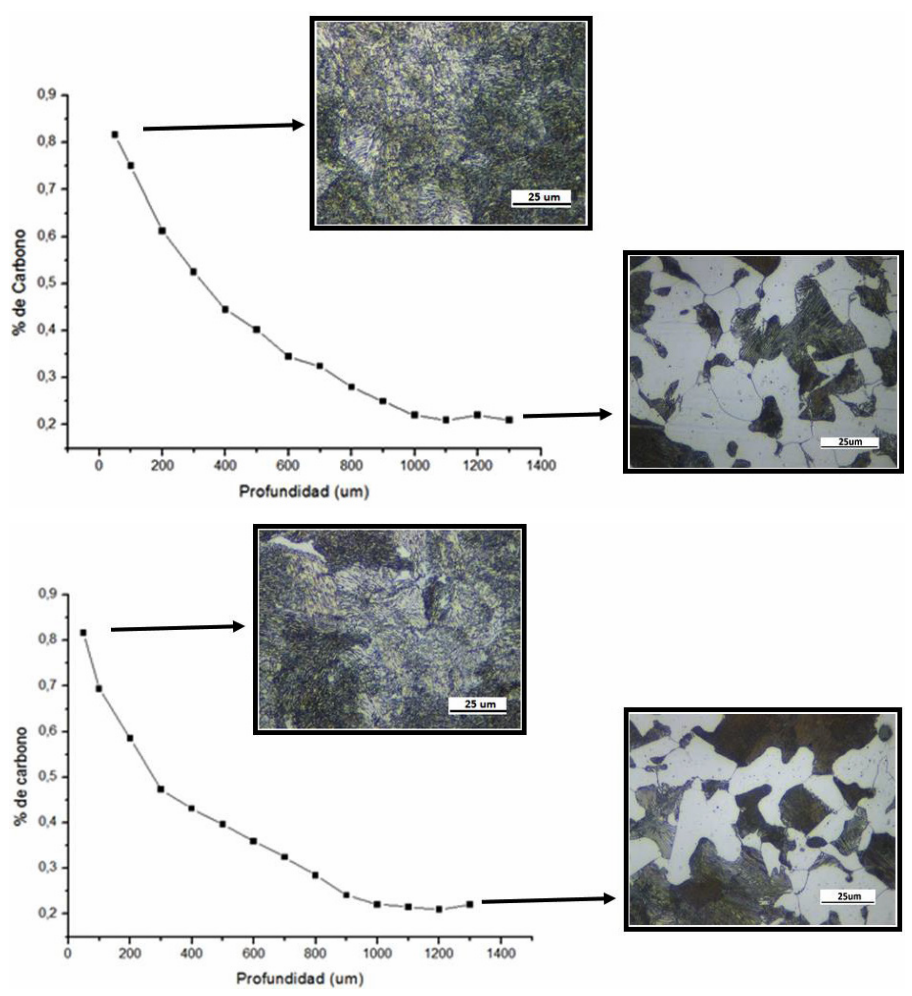

Figura 3. Perfiles de carbono en la capa carburizada para los aceros A1 y A2. Micrografías ópticas de la superficie y centro de ambos aceros. Ataque con Nital 2\%.
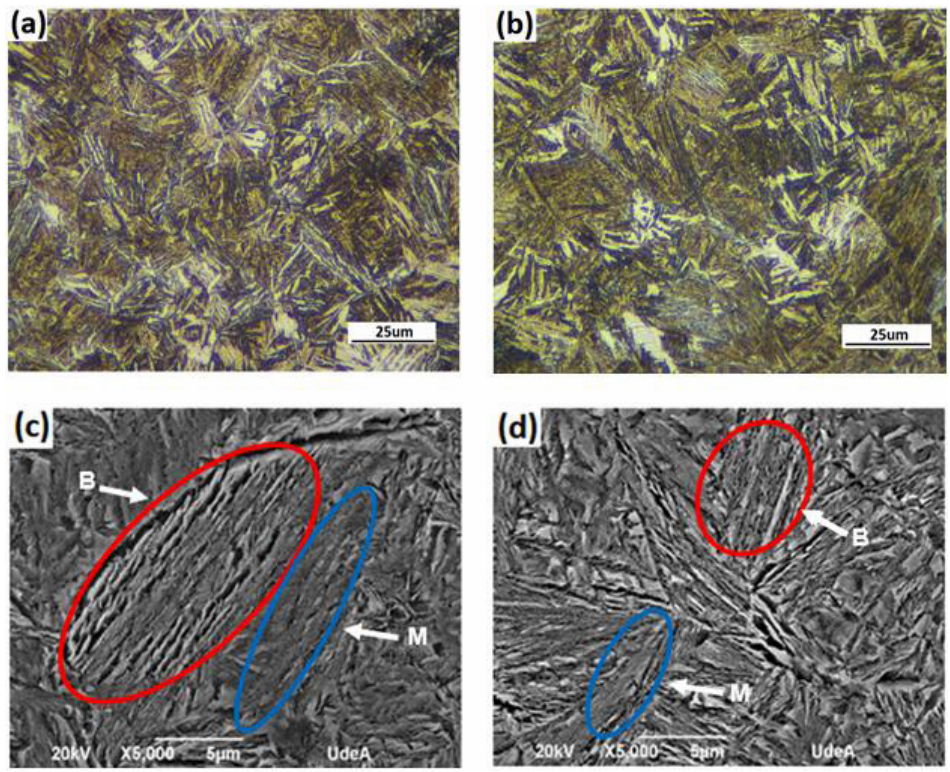

Figura 4. Micrografías ópticas y electrónicas de barrido para la capa del acero A1 tratado isotérmicamente a $270{ }^{\circ} \mathrm{C}$ : (a y c) 4 horas de sostenimiento y (b y d) 16 horas de sostenimiento. Donde, B: bainita y M: martensita. Ataque con Nital 2\%. 

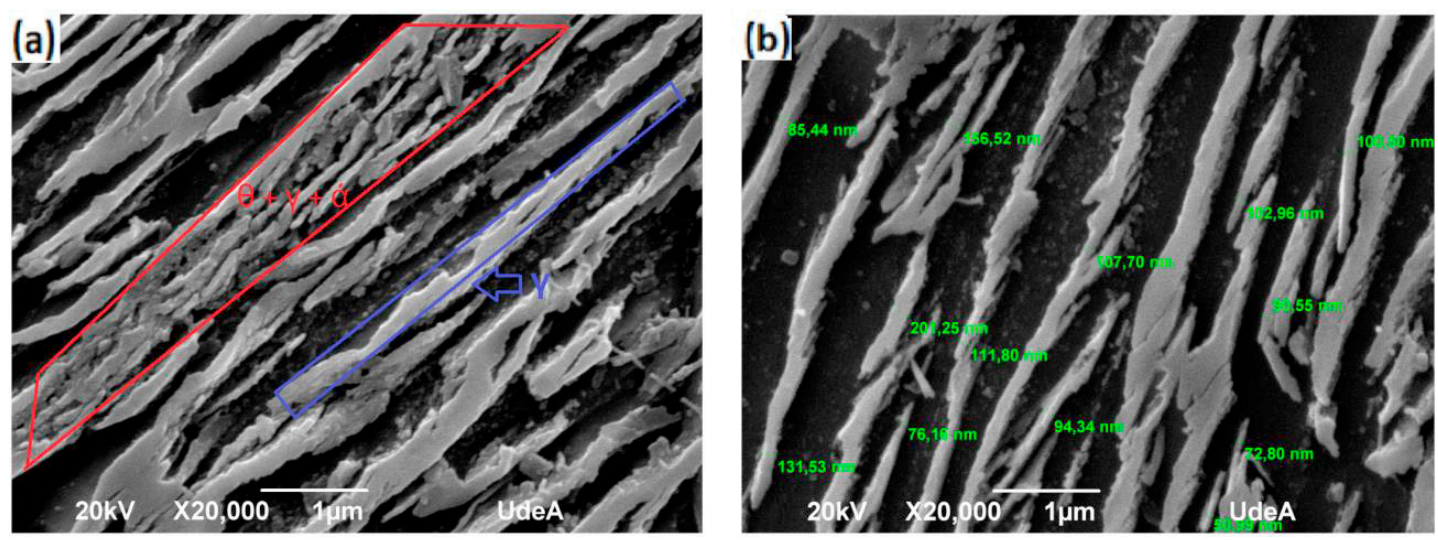

Figura 5. Micrografías electrónicas de barrido donde se presentan los espesores de separación entre las placas de ferrita bainítica de los aceros objeto de estudio: (a) A1 y (b) A2.

electrónica de barrido para establecer una mejor caracterización entre las fases presentes. Se pudo establecer que las placas de ferrita bainítica en el acero A1 están separadas por fronteras ligeramente desorientadas que pueden ser, dado el bajo contenido de silicio en este acero, partículas de cementita $(\theta)$ o placas de martensita $\left(\alpha^{\prime}\right)$ (ver Figura 5(a)). En la Figura 5(b), por otro lado, se aprecia que para el acero A2 las placas de ferrita bainítica están separadas, por lo que se presume son placas de austenita, dado el alto contenido de silicio en esta aleación [8], [12]. Estas placas de austenita tienen un espesor que varía entre $70 \mathrm{~nm}$ y $150 \mathrm{~nm}$. En ambos aceros se aprecia como las placas de ferrita bainítica crecen en grupos, normalmente denominados plumas. En cada pluma, se aprecia como las barras de ferrita son paralelas y de idéntica orientación cristalográfica.

A partir de la complejidad para identificar las fases presentes en el acero A2 mediante reactivo Nital, se procedió a utilizar reactivo Klemm 1. Ya que el contraste de color que ofrece este reactivo, permite de manera rápida y fácil, poder distinguir las diferentes fases dentro de la estructura objeto de estudio. En realidad, lo que sucede es que se genera una capa de depósito sobre la superficie del metal, donde el color que se observa por MO se genera como un efecto de interferencia entre el depósito y la superficie. En función de esto, y según el ASM Handbook [32], las fases que aparecen en las micrografías ópticas presentadas en las figuras 6 , se pueden definir de la siguiente manera: ferrita bainítica de color azul, martensita café y la austenita retenida en color blanco. En la Figura 6(a), se presenta una imagen obtenida por MO del acero A1, utilizando Klemm 1 como reactivo de ataque. Es evidente que el porcentaje de austenita retenida es poco, en comparación con el acero A2. El crecimiento de las placas de bainita es adifusional hasta un tamaño limitado e inmediatamente después el exceso de carbono difunde a la austenita residual [8]. En el caso del acero A1, en la austenita enriquecida se produce la precipitación de carburos de cementita, debido a que no existe el suficiente contenido de elementos inhibidores de la formación de carburos (e.g. silicio y aluminio).

En el caso del acero A2, utilizando Klemm 1 como reactivo de ataque (Figura 6 (b)), se observa que la ferrita bainítica posee una morfología en agujas característica de una bainita inferior, con un alto grado de refinamiento, morfología que se puede explicar como producto del alto contenido de carbono. Además, al existir un contenido de silicio $>1,5$ (\% wt.), se logra favorecer un pico de concentración en los inicios de la trasformación de la cementita, lo que produce un aumento local de la actividad del carbono [8]. Como consecuencia, el flujo de carbono es reducido y se inhibe el desarrollo de los embriones de cementita para esta aleación, logrando de esta manera estabilizar la austenita residual a temperatura ambiente, obteniéndose mayores contenidos de austenita retenida en la estructura final. De otro lado, las Figuras 6(c) y 6(d) muestran micrografías representativas de las zonas bainíticas obtenidas en el microscopio electrónico de barrido para el acero A2, en donde 

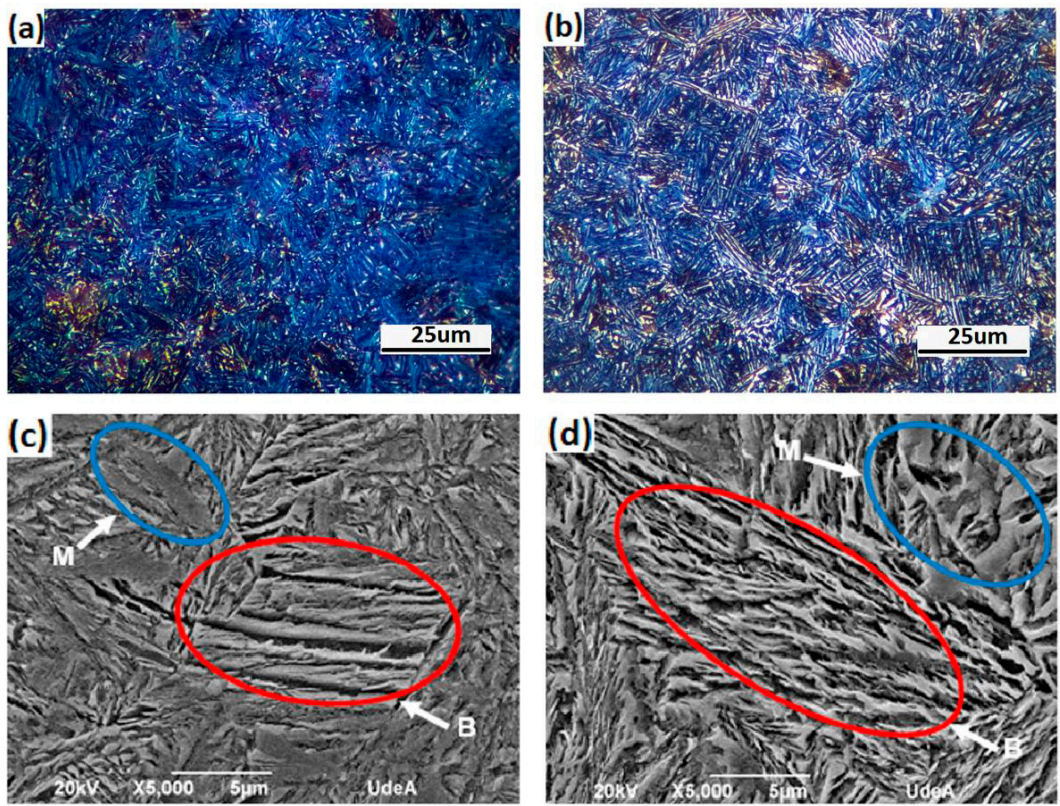

Figura 6. Micrografías ópticas y electrónicas de barrido para la capa carboaustemperada de los aceros: (a) $\mathrm{A} 1,270{ }^{\circ} \mathrm{C}$ - 16 horas, ataque con reactivo $\mathrm{Klemm} \mathrm{1,} \mathrm{(b)} \mathrm{A} 2,270{ }^{\circ} \mathrm{C}-16$ horas, ataque con reactivo Klemm 1, (c) A2, $270{ }^{\circ} \mathrm{C}-4$ horas, ataque con reactivo Nital $2 \%$ y (d) $\mathrm{A} 2,270{ }^{\circ} \mathrm{C}-16$ horas, ataque con reactivo Nital $2 \%$. Donde, B: bainita y M: martensita.

se observa la presencia de ferrita bainítica (B) y austenita retenida, además, de algunas zonas con presencia de martensita $(\mathrm{M})$.

En la Tabla 3 se muestran los resultados de la cuantificación de la fracción volumétrica por el método de conteo de puntos de la bainita y la martensita en la capa carbo-austemperada para los aceros A1 y A2. A partir de los análisis de DRX realizados en los dos aceros objeto de estudio, se pudieron identificar los picos característicos de la austenita retenida y de la ferrita (que puede ser ferrita bainítica, ferrita proeutectoide o martensita). Con el análisis de las intensidades fue posible determinar la fracción volumétrica de austenita retenida para ambos aceros.

Cabe destacar en primer lugar, que no se encontró ferrita proeutectoide ni perlita en ningún caso. A un tiempo de 4 horas de sostenimiento, la transformación bainítica ha logrado avanzar poco, por lo tanto, la microestructura es mayoritariamente martensítica, tras el temple a temperatura ambiente. De otra parte, a las 16 horas de transformación isotérmica se favorece una mayor fracción de bainita en detrimento de la martensita, por lo que la microestructura consta de ferrita bainítica, martensita y austenita retenida y/o carburos de cementita [33]. En el acero A2, las

Tabla 3. Cuantificación de las fases para los aceros A1 y A2 en función del tiempo de sostenimiento.

\begin{tabular}{|c|c|c|c|c|}
\hline \multirow{2}{*}{ Acero } & $\begin{array}{c}\text { Tiempo de } \\
\text { Sostenimiento (Horas) }\end{array}$ & $\begin{array}{c}\text { Fracción de Bainita } \\
(\boldsymbol{\%})\end{array}$ & $\begin{array}{c}\text { Fracción de Martensita } \\
\mathbf{( \% )}\end{array}$ & $\begin{array}{c}\text { Fracción de Austenita Retenida } \\
(\boldsymbol{\%})\end{array}$ \\
\hline \multirow{2}{*}{ A1 } & 4 & 35 & 62 & 3 \\
\cline { 2 - 5 } & 16 & 50 & 49,5 & 0,5 \\
\hline \multirow{2}{*}{ A2 } & 4 & 45 & 51 & 4 \\
\cline { 2 - 5 } & 16 & 70 & 22 & 8 \\
\hline
\end{tabular}


austenita cercana a la ferrita bainítica estará enriquecida en carbono, por lo que tras el temple a temperatura ambiente se tendrá una mezcla de bainita/martensita/ austenita retenida. Además, la fracción de austenita retenida es mayor en el acero A2 debido a su mayor concentración de silicio, que como se mencionó anteriormente inhibe la precipitación de carburos de cementita durante la reacción bainítica [27].

La microestructura para la condición A2/16h es mayoritariamente bainítica, con un porcentaje del 70\%, seguida por la presencia de martensita. Estos resultados son positivos, debido a que se denota también la presencia de austenita retenida, y al mismo tiempo, en función de la morfología laminar, es de esperar que se encuentre enriquecida en carbono. La baja fracción de volumen de austenita retenida obtenida en el acero A1, se debe a la presencia de carburos de cementita entre las lajas de ferrita bainítica, los cuales evitan la estabilización térmica y mecánica de la austenita a temperatura ambiente [8-10]. En el acero A2 la fracción de austenita retenida presente en las muestras se encuentra presente en dos morfologías: (1) entre las placas de ferrita bainítica en forma de láminas y (2) en forma de bloques (Figura 7). Estos bloques aislados de austenita retenida pueden afectar de un modo desfavorable el alargamiento. Esto se debe a que los bloques de austenita retenida se encuentran menos enriquecidos en carbono que las láminas, lo que los hacen más inestables mecánicamente, susceptibles de transformar a martensita no revenida mediante efecto TRIP [8].
En la Figura 8 se muestra la evolución de la microdureza en función de la profundidad de la capa carbo-austemperada para ambos aceros. En el caso del acero A1 se observa una máxima dureza en superficie de $725 \mathrm{HV}$ para 4 horas de sostenimiento y $748 \mathrm{HV}$ para 16 horas. Por otra parte, el acero A2 alcanzó una dureza en superficie de 550HV con 4 horas de sostenimiento y $575 \mathrm{HV}$ con 16 horas. La desaparición progresiva de la martensita, en el acero A2, es una de las causas en la diferencia de los niveles de dureza con respeto al acero A1. De otro lado, la presencia de carburos en la microestructura del acero A1, favorece un incremento notable en los valores de dureza logrados [9]. Sin embargo, las muestras del acero A2 pueden conducir a una mejor combinación de resistencia y ductilidad. Esto se debe, principalmente, a que la bainita posee un tamaño de grano muy fino que aporta una resistencia adicional, mientras que la presencia de austenita retenida en forma de láminas, contribuye, por su parte, a una mejor ductilidad [33]. Las láminas de austenita retenida también aportan una elevada tenacidad al impacto a las microestructuras bainíticas, contrario al efecto de los carburos en el acero A1 [34]. Cuantitativamente, la austenita retenida puede afectar a la resistencia de diversos modos. Por un lado, la austenita residual, durante el enfriamiento a temperatura ambiente, puede transformar a martensita aumentando la resistencia. Por otro lado, las láminas situadas entre las placas de ferrita bainítica pueden aumentar la resistencia del material mediante transformación a martensita durante el servicio, del mismo modo que ocurre con los aceros TRIP.
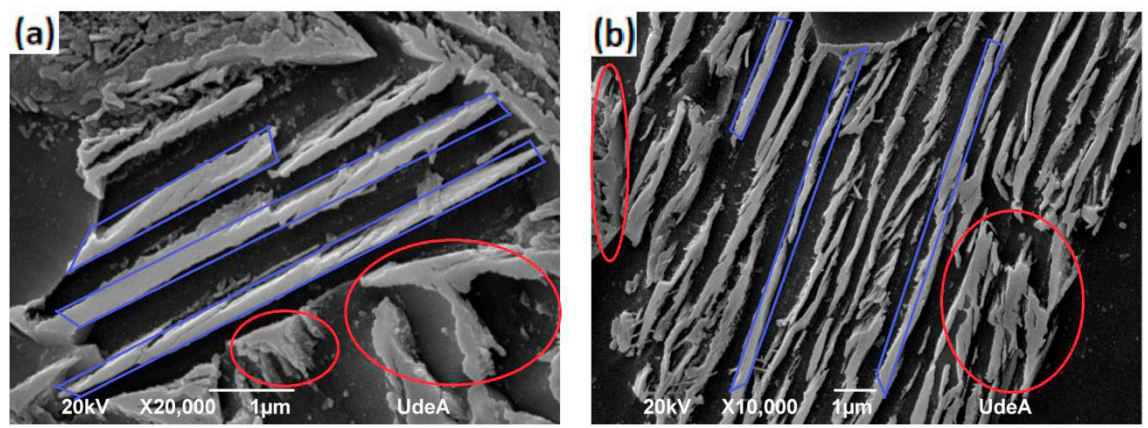

Figura 7. Micrografía electrónica de barrido donde se presentan las morfologías de la austenita retenida presentes en el acero A2 a diferentes tiempos de sostenimiento isotérmico: (a) 4 horas y (b) 16 horas. Dónde: rojo austenita en bloques y azul austenita en láminas. 


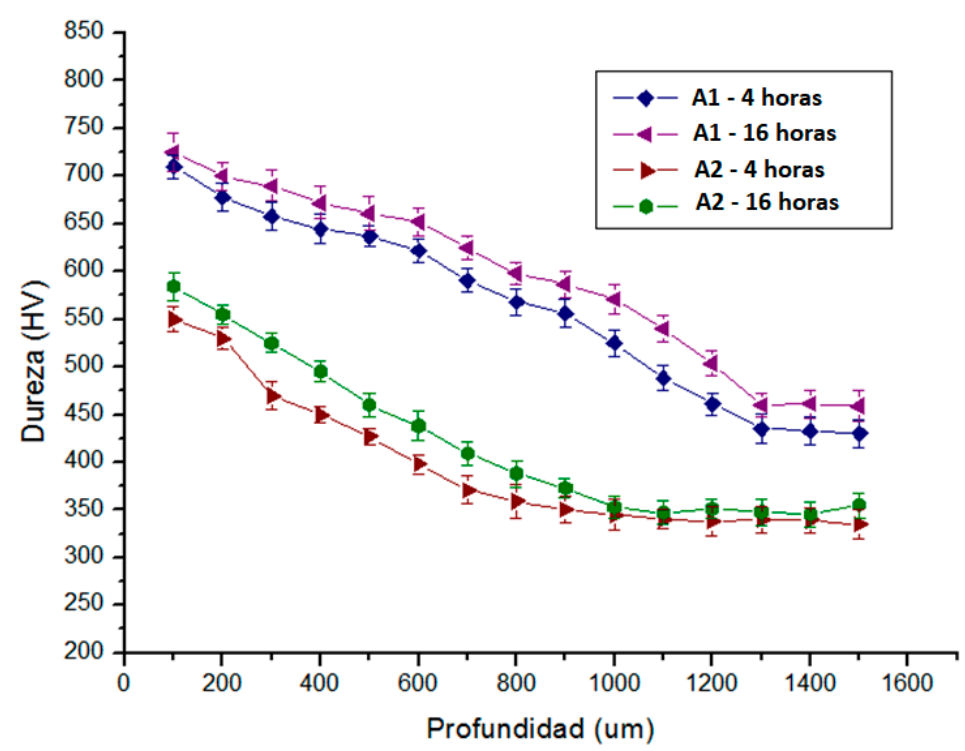

Figura 8. Perfil de microdureza para los aceros desarrollados.

De otro lado, la Figura 9 muestra las micrografías ópticas representativas del núcleo en los aceros A1 y A2. En las imágenes se observa la presencia de ferrita proeutectoide $\left(\alpha_{\text {pro }}\right)$, bainita (B) y martensita (M). La ferrita proeutectoide en la microestructura se explica por la austenización a $840{ }^{\circ} \mathrm{C}$, en la cual se logró generar una austenización completa para la superficie del acero e intercrítica para el núcleo, en función del contenido de carbono. La martensita se forma a partir de la austenita de alta
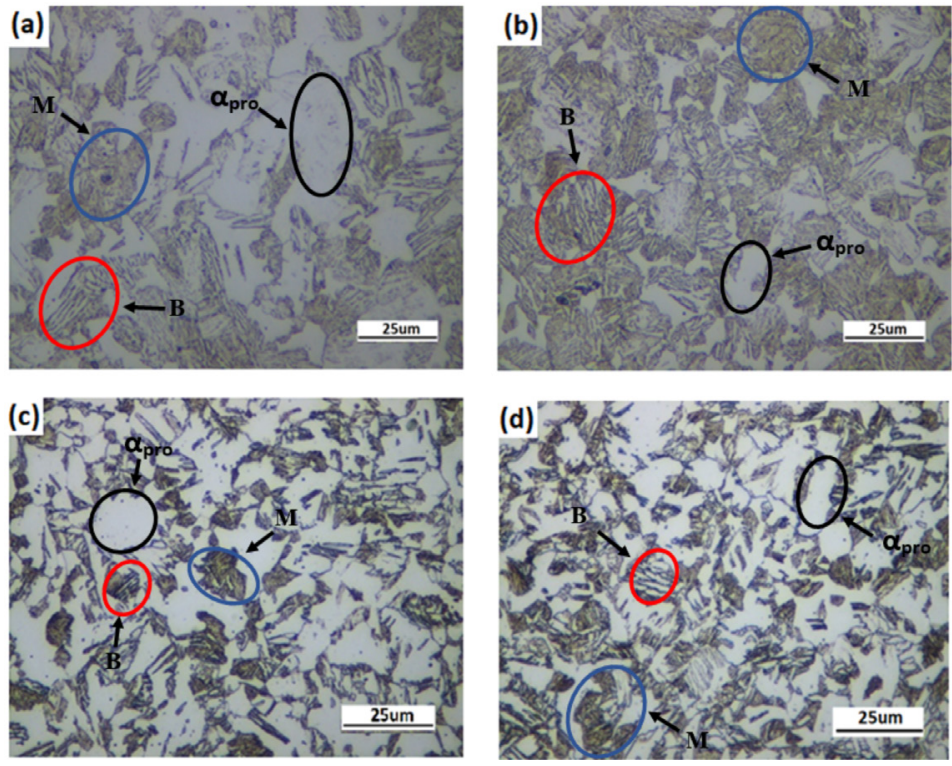

Figura 9. Micrografías ópticas del núcleo austemperado a $270{ }^{\circ} \mathrm{C}$ para A1: (a) 4 horas y (b) 16 horas y para A2: (c) 4 horas y (d) 16 horas. Dónde: M: martensita, B: bainita y apro: ferrita proeutectoide. Ataque con Nital 2\%. 
temperatura que no alcanzó a transformase durante el tratamiento isotérmico y que tampoco alcanzó a enriquecerse lo suficiente en carbono para ser estable a temperatura ambiente. Finalmente, la bainita es producto de la transformación isotérmica, el tamaño submicrométrico de las placas de ferrita bainítica y austenita retenida se debe a la temperatura de la transformación [35]. Esta estructura es prometedora para el núcleo de un acero carbo-austemperado, debido a la presencia de una matriz ferrítica y la capacidad de la austenita retenida de transformarse a martensita ante la aplicación de esfuerzos/ deformaciones externas, lo que contribuye con un mejoramiento de los niveles de ductilidad, lo cual a su vez aumenta el coeficiente de endurecimiento por deformación. La alta resistencia mecánica se deriva de la presencia de las fases de la transformación austenítica (bainita y martensita), las cuales actúan como un refuerzo de la matriz.

\section{CONCLUSIONES}

Es importante resaltar los siguientes aspectos: (1) La aleación del acero A2 con mayor cantidad de silicio ayudó a eliminar la precipitación de cementita entre las placas de ferrita bainítica, que sería muy perjudicial para la tenacidad, (2) la ruta de homogenización garantizó la obtención de un tamaño de grano fino y la eliminación de gradientes de concentración química, producto del proceso de solidificación y (3) Se evidenció la importancia del control de las variables de tratamiento de carboaustemperado, ya que a partir de estas se logra obtener microestructuras controladas en la capa y en el núcleo. Las condiciones de austenización permitieron obtener $100 \%$ de austenita de alta temperatura en la capa y $\sim 30 \%$ para la región del núcleo, lo que garantiza durante el tratamiento isotérmico obtener estructuras bainítica con su máxima fracción, en tiempos de austemperado no muy prolongados, para la superficie, mientras en el núcleo se obtienen microestructuras multifásicas, compuesta por ferrita proeutectoide, martensita y bainita. A partir de dichos resultados se podría esperar un mejor balance entre los niveles de resistencia en la capa carbo-austemperada y los niveles de tenacidad en el núcleo.

La utilización de silicio como elemento de aleación en los aceros A1 y A2, jugo un rol importante en las características microestructurales y mecánicas obtenidas. El silicio al disolverse completamente en la ferrita, favorece un incremento de la resistencia sin afectar la ductilidad, favorece los niveles de dureza, la resistencia al desgaste, el límite elástico y los esfuerzos de fluencia. Además, este elemento permite mejorar los niveles de endurecimiento, cuando se aplican tratamientos térmicos superficiales, como el de carburización.

Finalmente, es bueno resaltar, que se logró experimentalmente demostrar que las herramientas teóricas basadas en principios cinéticos y de transformaciones de fase en estado sólido, ya existentes, programa MAP_STEEL_MUCG83, se pueden emplear con éxito en el diseño de aceros carbo-austemperados a partir del cálculo de los diagramas TTT.

\section{REFERENCIAS}

[1] K. Hayrynen, K. Brandenberg and J. Keough. "Carbo-Austempering TM - A New Wrinkle?'. Applied Process Inc. Technologies Div. Livonia, Michigan, USA. 2002. DOI: 10.4271/2002-01-1478.

[2] F. Caballero, M. Miller and C. Garcia-Mateo. "Influence of transformation temperature on carbide precipitation sequence during lower bainite formation". Mater. Chem. Phys. Vol. 146, Issue 1-2, pp. 50-57. 2014.

[3] J. Keough. "Austempering: A Small, Niche Heat Treatment With Large Powertrain Implications". Technical Update: Gear Industry Journal. Applied Process Inc. Technologies Div. Livonia, Michigan, USA, pp. 42-43. 2004.

[4] F. Zhang, T. Wang, P. Zhang, B. Zheng, Lv. Zhang and Y. Zheng. "A novel method for the development of a low-temperature bainitic microstructure in the surface layer of low-carbon steel". Scr. Mater. Vol. 59, Issue 3, pp. 294-296. 2008.

[5] Y. Wang, Z. Yang, F. Zhang and D. Wu. "Microstructures and mechanical properties of surface and center of carburizing 23Cr2Ni2Si1Mo steel subjected to lowtemperature austempering". Mater. Sci. Eng. A. Vol. 670, pp. 166-177. July, 2016.

[6] P. Zhang, F. Zhang, Z. Yan, T. Wang and L. Qian. "Rolling Contact Fatigue of a Low Temperature Bainite in Surface Layer of 
a Low Carbon Steel". Mater. Sci. Forum. Vol. 675-677, pp. 585-588. 2011.

[7] E. Skołek, K. Wasiak and W. Świątnicki. "Structure and properties of the carburised surface layer on 35CrSiMn5-5-4 steel after nanostructurization treatment". Mater. Tehnol. Vol. 49, Issue 6, pp. 933-939. 2015.

[8] M. Santofimia. "La transformación bainítica sin formación de carburos en aceros". Tesis, (Doctor en ciencias físicas), España, Universidad Complutense de Madrid, p. 198. 2007. URL: http://eprints.ucm.es/7460/

[9] H. Bhadeshia and D. Edmonds. "Bainite in Silicon Steels: A New Composition-Property approach". Part II. Met. Sci. Vol. 17, pp. 420425. 1983.

[10] F. Caballero, H. Bhadeshia, D. Jones, P. Brown and K. Mawella. "Diseño de nuevos aceros bainíticos". Rev. Metal. Madrid. Vol. 38, pp. 3-13. 2002.

[11] J. Cornide. "Aceros bainiticos avanzados: Mecanismos de transformacion y propiedades mecanicas". Doctor en ciencias físicas, España, Universidad Complutense de Madrid, p. 184. 2012.

[12] H. Bhadeshia. "Bainite in steels: Transformations, Microstructure and Properties". 2nd ed. p. 454. 2001.

[13] Z. Yang and H. Fang. "An overview on bainite formation in steels". Curr. Opin. Solid State Mater. Sci. Vol. 9, Issue 6, pp. 277-286. 2005.

[14] Springer, Advanced Steels. "The recent Scenario in Steel Science and Technology". Metallurgical Industry Press - Springer. 2011.

[15] A. Valencia. "Los aceros avanzados-Advanced Steels". Revista Colombiana de Materiales, $\mathrm{N}^{\circ}$ 2, pp. 1-13. 2012.

[16] WorldAutoSteel. "Evolving AHSS Types." URL: http://www.worldautosteel.org/ steel-basics/steel-types/evolving-ahss-types/

[17] C. Garcia-Mateo, T. Sourmail, F. Caballero, V. Smanio, M. Kuntz, C. Ziegler, A. Leiro, R.E. Vuorinen Elvira and T. Teeri. "Nanostructured steel industrialization: a plausible reality". Materials Science and Technology. Vol. 30, pp. 1071-1078. 2014.

[18] C. Garcia-Mateo and F. Cabllero. "Understanding the mechanical properties of nanostructured bainite". Handbook of Mechanical Nanostructuring, Chapter 3, pp. 35-65. 2015.
[19] C. Garcia-Mateo, G. Paul, C. Somani, D. Porter, L. Bracke, A. Latz, C. Garcia de Andres and F. Caballero. "Transferring Nanoscale Bainite Concept to Lower C Contents: A Perspective". Metals (Basel). Vol. 7, p. 159. 2017.

[20] F. Caballero, M. Miller y C. Garcia-Mateo. "NANOBAIN-Aceros bainíticos avanzados. Un análisis a nivel atómico". X Congreso Nacional de Materiales (Donostia - San Sebastián, pp. 18-20. Junio 2008.

[21] C. García-Mateo, F. Caballero y H. Bhadeshia. "Superbainita. Una nueva microestructura bainítica de alta resistencia". Rev. Metal. Vol. LIX N ${ }^{\circ}$ C, pp. 121-129. 2001.

[22] M. Peet and H. Bhadeshia. Program MAP STEEL_MUCG83, Cambridge, Materials Algorithms Project. URL: http://www.msm. cam.ac.uk/map/steel/programs/mucg83.html

[23] A. Gorni. "Steel Forming and Heat Treating Handbook". São Vicente, Brazil, No January, p. 206. 2018. URL: http://www.gorni.eng. br/e/Gorni_SFHTHandbook.pdf

[24] American society for testing and materials (ASTM). "E3-11: Standard Guide for Preparation of Metallographic Specimens 1". ASTM Int. Vol. I, pp. 1-12. 2011.

[25] American society for testing and materials (ASTM). "Standard test methods for determining average grain size". E112-13. ASTM Int., pp. 1-28. 2013.

[26] American society for testing and materials (ASTM). "Standard Test Method for Determining Volume Fraction by Systematic Manual Point Count". D562 - 11. Annu. B. ASTM Stand., N ${ }^{\circ}$ C, pp. 1-7. 2011.

[27] L. Morales-Rivas, F. Caballero and C. GarciaMateo. "Retained Austenite: Stability in a Nanostructured Bainitic Steel". Encycl. Iron, Steel, Their Alloy, pp. 3077-3087. 2016.

[28] M. Sherif. "Characterisation and Development of Nanostructured, Ultrahigh Strength, and Ductile Bainitic Steels". Doctoral thesis. 2006. URL: https://doi.org/10.17863/ CAM.14211

[29] C. Hofer, F. Winkelhofer, H. Clemens and S. Primig. "Morphology change of retained austenite during austempering of carbide-free bainitic steel”. Mater. Sci. Eng. A. Vol. 664, pp. 236-246. 2016. 
[30] American society for testing and materials (ASTM). "Standard Test Methods for Vickers Hardness and Knoop Hardness of Metallic". Am. Soc. Test. Mater. Vol. 82, Issue July, pp. 1-27. 2016.

[31] A. Valencia. "Tecnologia del tratamiento térmico de los metales". Editorial Universidad de Antioquia, XVIII. Colección Ciencia y Tecnología, Medellin, p. 632. 2009.

[32] G. Vander Voort. "ASM Handbook: Metallography and microestructures". Vol. 9, p. 257. 1985.
[33] C. Garcia-Mateo and F. Caballero. "Nanocrystalline Bainitic Steels for Industrial Applications". Nanoenabled Materials and Coatings for Energy Applications, pp. 707724. 2017.

[34] C. Garcia-Mateo and F. Cabllero. "Understanding the mechanical properties of nanostructured bainite". Handbook of Mechanical Nanostructuring, pp. 35-65. 2015.

[35] R. Aristizábal. "Intercritical Heat Treatments in Ductile Iron and Steel". Dissertation Research. Thesis of D.Sc. University of Alabama at Birmingham. 2012. 